\title{
The Application of EMMS Model to Plane Jet
}

\author{
Ke-Zhi YU ${ }^{a^{*}}$, Yan-Ling LIU ${ }^{b}$ and Hai ZHANG ${ }^{c}$ \\ Department of Refrigeration Engineering, Shanghai Ocean University, Shanghai 201306, China \\ akzyu@shou.edu.cn bylliu@shou.edu.cn, cheyhai@126.com \\ *Corresponding author
}

Keywords: Energy-minimization multi-scale, Plane jet, Dissipation, Gauss distribution.

\begin{abstract}
The energy minimization multi-scale model was applied to the plane jet. The stability condition of plane jet was adopted to predict the velocity distribution of plane jet. .The ratio of total dissipation to viscous dissipation tends to the maximum was used as the optimization condition. The simulation shows that the Gauss velocity distribution can be obtained in the plane jet when the ratio of jet section semi thickness to the jet distance is considered as a constant.
\end{abstract}

\section{Introduction}

Multi-scale EMMS (Energy-minimization) model is a mechanism model, which is widely used in gas-solid two-phase flow, and has achieved great success [1-4]. Li Jinghai eatl. found that the coordination mechanism between the inertial and viscous effect in single-phase turbulence is very similar to that in a gas-solid system. Therefore, the EMMS model can be extended to turbulence field and the turbulence stability conditions is proposed. In this model, the shear viscous dissipation rate tend toward minimum and the total dissipation tend to maximum at the same time. This model predicted the turbulent pipe flow velocity distribution successfully [5, 6].

Jet flow is a common flow besides pipe flow. Three methods are often used for the analysis of turbulent flow. (1) The solution of integral equation. Gaussian distribution of jet velocity of cross section is usually adopted [7]. (2) Differential equations include W.Tollmienn theoretical solution [8] and Görtler theoretical solution [9]. (3) Numerical simulation solution. These methods involve urbulent model and calculation method. This paper attempts to apply the energy minimization multi-scale (EMMS) model to the plane jet, to predict the velocity distribution of the plane jet.

\section{Assumed Velocity Distribution in a Plane Jet}

Plane jet can be divided into three sections [7]: initial segment, transition segment and main segment (Fig.1)

In the fully developed region of the jet flow in the main section, the velocity distribution of each section is similar [7].

$$
\frac{u}{u_{m}}=f\left(\frac{y}{b}\right)
$$

Where $b$ is the characteristic half thickness of the jet cross section.

Based on the experimental results and the random nature of the turbulence, the assumption of reasonable velocity distribution:

$$
u=u_{m} e^{-\frac{y^{n}}{b^{n}}}
$$

Where $n$ is an integer variable to be determined. 


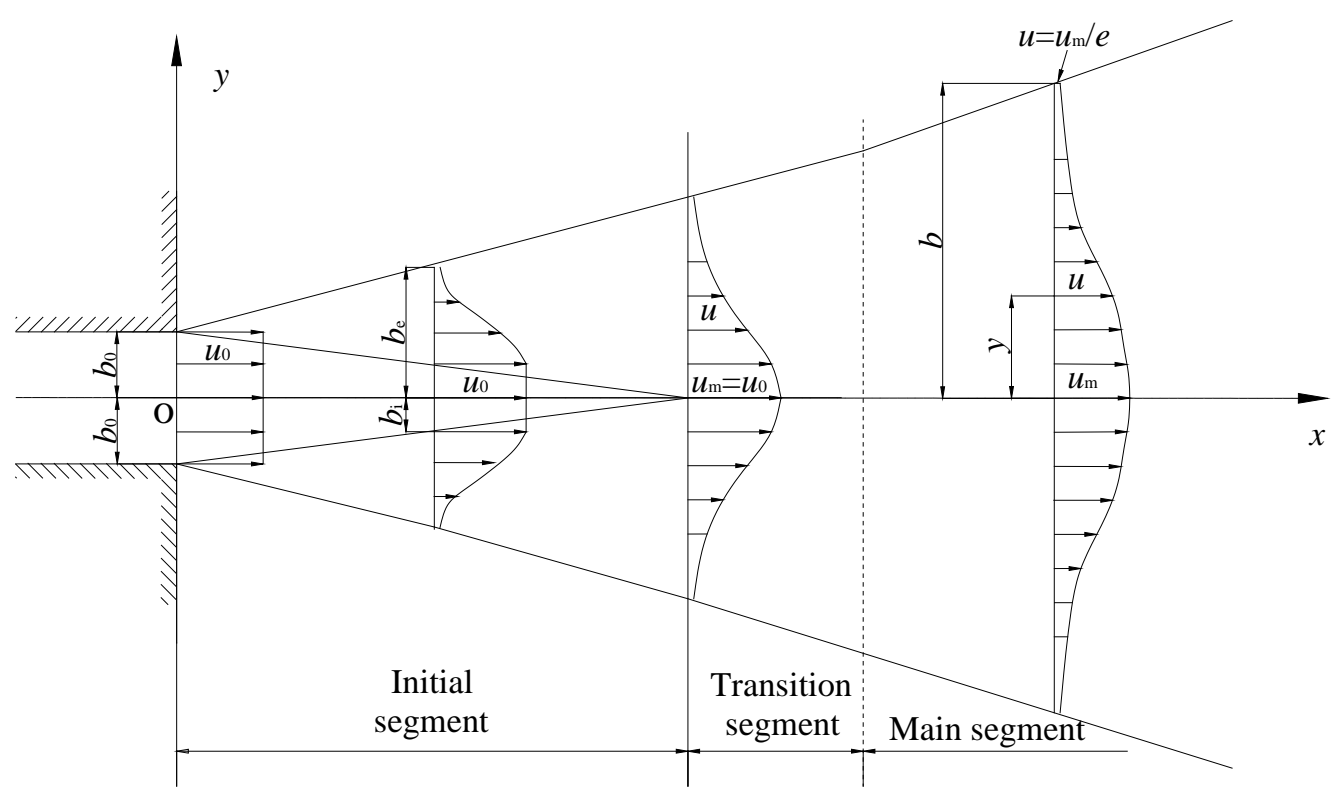

Fig.1 Plane jet

\section{The Basic Characteristics of Plane Jet}

Kinetic energy of arbitrary cross section. Kinetic energy

$$
E=\int_{-\infty}^{\infty} \rho u \cdot \frac{u^{2}}{2} d y=\rho b u_{m}^{3} \int_{0}^{\infty} e^{-3 \eta^{n}} d \eta=\rho b u_{m}^{3} e(n)
$$

where $e(n)=\int_{0}^{\infty} e^{-3 \eta^{n}} d \eta$, is a function of the variable $\mathrm{n}$, as shown in Table 1 .

Table 1 The relationship between $n$ and $e(n), m(n)$ and $w(n)$

\begin{tabular}{cccc}
\hline$n$ & $e(n)$ & $m(n)$ & $w(n)$ \\
\hline 1 & 0.33333 & 0.5 & 0.5 \\
2 & 0.511663 & 0.626657 & 0.156664 \\
3 & 0.619157 & 0.708758 & 0.0947823 \\
4 & 0.688717 & 0.762191 & 0.0683097 \\
5 & 0.737052 & 0.799312 & 0.053494 \\
6 & 0.772496 & 0.826504 & 0.0439938 \\
7 & 0.799567 & 0.847248 & 0.0373734 \\
8 & 0.820903 & 0.863582 & 0.0324919 \\
\hline
\end{tabular}

The change of kinetic energy along the flow direction

$$
\frac{d E}{d x}=\rho \pi e(n) \frac{d\left(b u_{m}^{3}\right)}{d x}
$$

Momentum of arbitrary cross section. Jet momentum

$$
M=\int_{-\infty}^{\infty} \rho u^{2} d y=2 \rho b u_{m}{ }^{2} \int_{0}^{\infty} e^{-2 \eta^{n}} d \eta=2 \rho b u_{m}{ }^{2} m(n)
$$


where $m(n)=\int_{0}^{\infty} e^{-2 \eta^{n}} d \eta$, is a function of the variable $n$, as shown in Table 1 .

The momentum of each section along the jet is equal to the momentum of the exit $M_{0}=2 \rho u_{0}^{2} b_{0}$ where $u_{0}, b_{0}$ is the jet exit velocity and the half width.

Therefore

$$
b u_{m}^{2} m(n)=b_{0} u_{0}^{2}
$$

The relationship among parameters. For a plane jet [7]'

$$
\begin{aligned}
& \frac{u_{m}}{u_{0}}=a \sqrt{\frac{2 b_{0}}{x}} \\
& b=\varepsilon x
\end{aligned}
$$

where $a$ and $\varepsilon$ is constant, according to the Equation (7), the relationship between the two parameters is

$$
a^{2} \varepsilon=\frac{1}{2 m(n)}
$$

\section{Stability Analysis of Plane Turbulent Jet Flow}

Stability condition. In a plane jet, the local shear dissipation of unit volume can be expressed as [10]

$$
W_{v}(y)=\mu\left(\frac{d u}{d y}\right)^{2}
$$

Its integral over the whole jet section

$$
\overline{W_{v}}=\int_{-\infty}^{\infty} W_{v} d y=\frac{2 n^{2} \mu u_{m}^{2}}{b} \int_{0}^{\infty} \eta^{2 n-2} e^{-2 \eta^{n}} d \eta=\frac{2 n^{2} \mu u_{m}^{2}}{b} w(n)
$$

where $w(n)=\int_{0}^{\infty} \eta^{2 n-2} e^{-2 \eta^{n}} d \eta$, is a function of the variable $n$, as shown in Table 1

Accordingly, in a plane jet, the total energy consumption of the unit volume can be considered as the attenuation of the kinetic energy of the jet, namely

$$
\overline{W_{T}}=-\frac{d E}{d x}=-\frac{d\left[\rho b u_{m}^{3} e(n)\right]}{d x}=-\rho e(n) b u_{m}^{2} \frac{d u_{m}}{d x}
$$

According to the stability condition of the turbulent flow $[5,6]$ :

$$
\left.\overline{W_{v}} \rightarrow \min \right|_{\overline{W_{t e}} \rightarrow \max }
$$

Where $\overline{W_{t e}}$ is caused by the inertia of the turbulent pulse dissipation, expressed as:

$$
\overline{W_{t e}}=\overline{W_{T}}-\overline{W_{v}}
$$


Stability condition transformation. It is a double peak value problem in Eq.13. As the ratio of a maximum value to a minimum value is a maximum value, the double peak value problem also can be expressed as a single target extremal optimization problem:

$$
G=\frac{\overline{W_{t e}}}{\overline{W_{v}}}=\frac{\overline{W_{T}}-\overline{W_{v}}}{\overline{W_{v}}}=\frac{\overline{W_{T}}}{\overline{W_{v}}}-1 \rightarrow \max
$$

The results based on Eq.15 can be analyzed in order to obtain a reasonable velocity distribution of plane jet flow.

\section{Results Analysis and Discussion}

Besides extreme condition based on Eq.15, which variable among $a$, and $\varepsilon$ in Eq. 9 can be considered as a constant, may leads to four different results. It is shown in Table 2, where $\operatorname{Re}=\frac{2 \rho u_{0} b_{0}}{\mu}$

Table 2 different extreme conditions and constants of plane jet

\begin{tabular}{ccc}
\hline & $a$ is a constant & $\varepsilon$ is a constant \\
$\overline{W_{T}}=-\rho e(n) b u_{m}^{2} \frac{d u_{m}}{d x}$ & $\frac{\rho e(n) a u_{0}^{3}}{2 m(n)} b_{0} \sqrt{\frac{2 b_{0}}{x^{3}}}$ & $\frac{\rho e(n) u_{0}^{3}}{2 \sqrt{\varepsilon} m^{3 / 2}(n)} b_{0} \sqrt{\frac{b_{0}}{x^{3}}}$ \\
$\overline{W_{v}}=\frac{2 n^{2} \mu u_{m}{ }^{2}}{b} w(n)$ & $\frac{8 n^{2} \mu u_{0}^{2} a^{4} b_{0} w(n) m(n)}{x^{2}}$ & $\frac{2 n^{2} \mu u_{0}^{2} b_{0} w(n)}{\varepsilon^{2} m(n) x^{2}}$ \\
$G=\frac{\overline{W_{T}}}{\overline{W_{v}}}-1$ & $\frac{\operatorname{Re} \sqrt{\frac{x}{16 a^{3}}} \frac{e(n)}{n^{2} m^{2}(n) w(n)}-1}{\frac{\sqrt{2} \varepsilon^{3 / 2} \operatorname{Re}}{8} \sqrt{\frac{x}{2 b_{0}}} \frac{e(n)}{n^{2} m^{1 / 2}(n) w(n)}-1}$ \\
\hline
\end{tabular}

$a$ is a constant. Objective function

$$
G=\frac{\operatorname{Re}}{16 a^{3}} \sqrt{\frac{x}{2 b_{0}}} \frac{e(n)}{n^{2} m^{2}(n) w(n)}-1
$$

The different variable values $n$ will lead to different $G$, which can be shown in Fig.2.

From Fig. 2, the increase of $n$ decreases $16 a^{3}(G+1) /\left(\operatorname{Re} \sqrt{\frac{x}{2 b_{0}}}\right)$, result in the reduction of $G$. It means that $n=1$ is the optimal value, which obviously does not meet the accepted Gauss distribution function. 


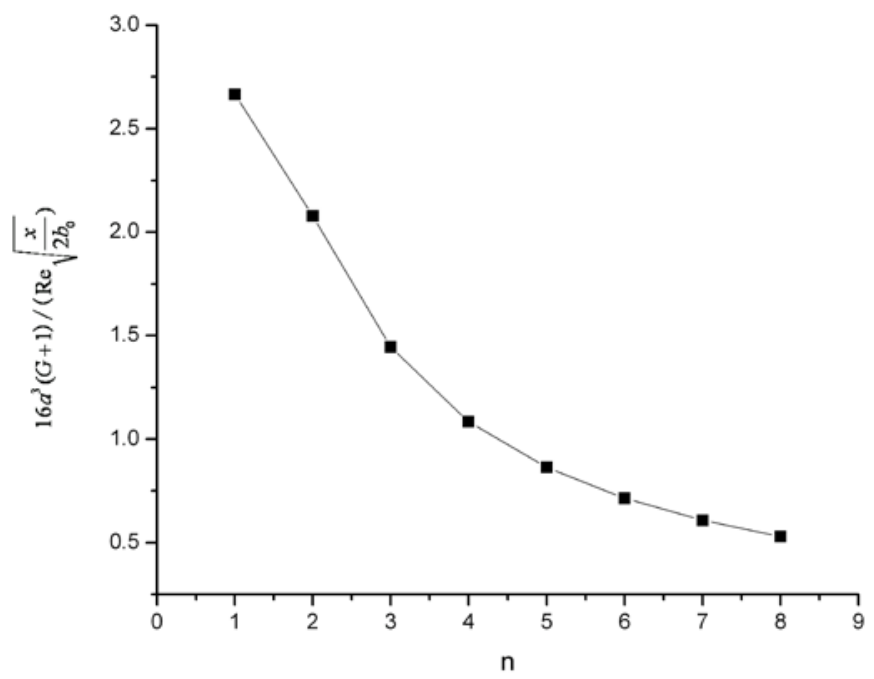

Fig. 2 The relationship between $\mathrm{n}$ and $16 a^{3}(G+1) /\left(\operatorname{Re} \sqrt{\frac{x}{2 b_{0}}}\right)$ when a is a constant

$\varepsilon$ is a constant. Objective function

$$
G=\frac{\sqrt{2} \varepsilon^{3 / 2} \operatorname{Re}}{8} \sqrt{\frac{x}{2 b_{0}}} \frac{e(n)}{n^{2} m^{1 / 2}(n) w(n)}-1
$$

The different variable values $n$ will lead to different $G$, which can be shown in Fig.3.

Fig.3 shows that $n$ obtain the optimal value 2 regardless of $\operatorname{Re} \sqrt{\frac{x}{2 b_{0}}}$, which meets the recognized Gauss distribution function.

Therefore, the optimal results can be obtained when $G$ is the objective function.

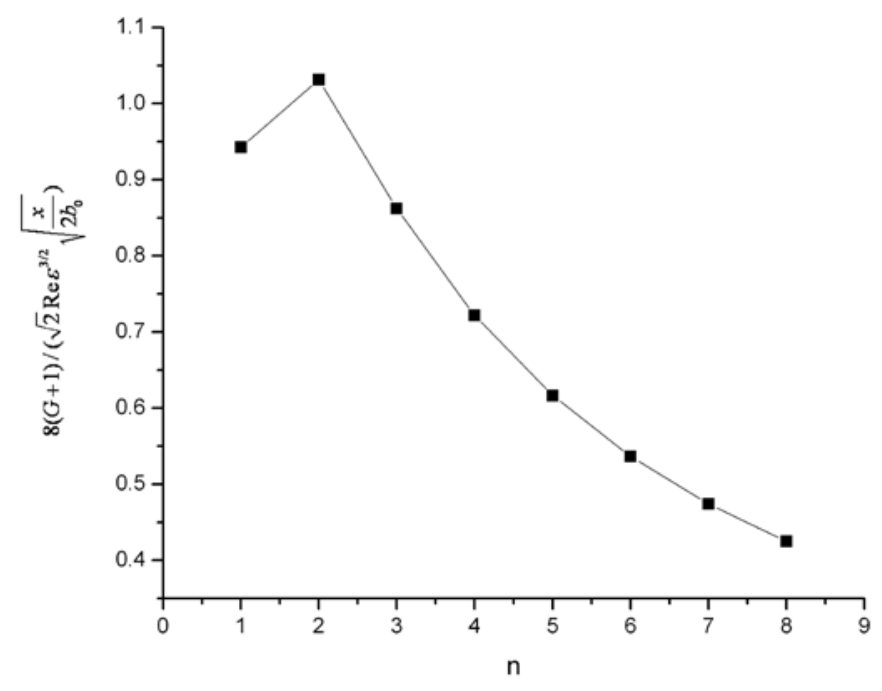

Fig. 3 Relationship between $8(G+1) /\left(\sqrt{2} \operatorname{Re} \varepsilon^{3 / 2} \sqrt{\frac{x}{2 b_{0}}}\right)$ and n when $\varepsilon$ is a constant

\section{Summary}

In this paper, the EMMS energy minimum scale model is applied to the study of the plane jet, and the flow velocity distribution is predicted by the stability condition of the planar jet. The main 
conclusions of the simulation are as follows:

(1) The stability condition of the plane jet can be transformed an optimization condition, in which the ratio of total dissipation to viscous dissipation tends to the maximum.

(2) When the ration of jet section semi thickness to the jet distance is considered as a constant, Gaussian distribution will be obtained for plane jet velocity profile.

\section{Acknowledgment}

This work was financially supported by Science and technology support program of Shanghai Science and Technology Commission (13dz1203002)

\section{References}

[1] Li Jinghai. The two-phase multi-scale model and energy minimization methods [M] Beijing: Institute of Chemical Metallurgy, 1987.

[2] Xu Guangwen The heterogeneous flow structure simulation in a circulating fluidized bed [M] Beijing: Institute of Chemical Metallurgy, 1996.

[3] Cheng Congli. Energy minimization multi-scale ring nuclear model in a circulating fluidized bed [M] Beijing: Institute of Process Engineering, 2001.

[4] Wang Linna The non-uniform gas-solid two phase flow and multiscale mass transfer model and experimental verification [M] Beijing: Institute of Process Engineering, 2002.

[5] Li Jinghai, Zhang Zhongdong, Ge Wei, Sun Qicheng, Yuan Jie. A simple variational criterion for turbulent flow in pipe [J]. Chemical Engineering Science, 1999, 54: 1151-1154.

[6] Li Jinghai, Zhang Zhongdong, Ge Wei, Yuan Jie. The extreme conditions in dissipative structures for two mechanisms coexistence [J] Chinese Science Bulletin, 1999, 44 (6): 613-617.

[7] Yu Changzhao, Turbulent jet [M] Beijing: High Education Press, 1993.

[8] Tollmien W., Berechnung turbulenter Ausbreitungsvorgänge,ZAMM,6(6),466,1926.

[9] Görter H., Berechnung von Aufgaben der freien Turbulenz auf Grund eines neuen Näherungsansatzes. ZAMM,22(5),1942

[10]Bejan A. Entropy generation through heat and fluid Flow [M]. New York: Wiley, 1982 